\title{
Population Pharmacokinetic and Exposure-Response Analysis of Finerenone: Insights Based on Phase Ilb Data and Simulations to Support Dose Selection for Pivotal Trials in Type 2 Diabetes with Chronic Kidney Disease
}

\author{
Nelleke Snelder ${ }^{1} \cdot$ Roland Heinig $^{2} \cdot$ Henk-Jan Drenth ${ }^{1} \cdot$ Amer Joseph $^{3} \cdot$ Peter Kolkhof ${ }^{4}$. Jörg Lippert ${ }^{5}$. \\ Dirk Garmann ${ }^{5} \cdot$ Bart Ploeger $^{6} \cdot$ Thomas Eissing $^{7}$
}

Published online: 4 October 2019

(c) The Author(s) 2019

\begin{abstract}
Background Finerenone (BAY 94-8862) is a potent non-steroidal, selective mineralocorticoid receptor antagonist being developed for the treatment of patients with type 2 diabetes and chronic kidney disease.

Methods We present the population pharmacokinetics and pharmacodynamics (PD) analysis for efficacy and safety markers based on data from two clinical phase IIb studies: ARTS-DN (NCT01874431) and ARTS-DN Japan (NCT01968668). Results The pharmacokinetics of finerenone were adequately characterized, with estimated glomerular filtration rate (eGFR) and body weight as influencing covariates. The area under the plasma concentration-time curve in Japanese patients did not differ from that in the global population, and the investigated pharmacokinetics were dose- and time-linear. In addition, the pharmacokinetic model provided robust individual exposure estimates to study exposure-response. The concentration-effect relationship over time for the efficacy marker urinary albumin:creatinine ratio (UACR) was well-characterized by a maximum effect model indicating saturation at high exposures. For the safety markers, a log-linear model and a power model were identified for serum potassium concentration and eGFR, respectively, indicating attenuation of effect gains at high exposures. There was no apparent ethnic effect on the investigated pharmacokinetic-pharmacodynamic relationships. The model-predicted times to reach the full (99\%) steady-state drug effect on UACR, serum potassium, and eGFR were 138,20 , and 85 days, respectively, while the pharmacokinetic half-life was 2-3 h and steady state was achieved after 2 days, indicating timescale separation.

Conclusion Our dose-exposure-response modeling and simulation indicates effects were largely saturated at finerenone $20 \mathrm{mg}$ and doses of both 10 and $20 \mathrm{mg}$ once daily appear safe and efficacious at reducing albuminuria.
\end{abstract}

Electronic supplementary material The online version of this article (https://doi.org/10.1007/s40262-019-00820-x) contains supplementary material, which is available to authorized users.

Thomas Eissing

thomas.eissing@bayer.com

1 Leiden Experts on Advanced Pharmacokinetics and Pharmacodynamics (LAP\&P), Leiden, The Netherlands

2 Clinical Pharmacology, Bayer AG, Wuppertal, Germany

3 Clinical Development Cardiology and Nephrology, Bayer AG, Berlin, Germany

4 Preclinical Research Cardiovascular, Bayer AG, Wuppertal, Germany

5 Clinical Pharmacometrics, Bayer AG, Wuppertal, Germany

6 Clinical Pharmacometrics, Bayer AG, Berlin, Germany

7 Clinical Pharmacometrics, Bayer AG, Leverkusen, Germany

\section{Key Points}

Population pharmacokinetic analysis of finerenone quantified estimated glomerular filtration rate (eGFR) and body weight, but not ethnicity, as significant covariates.

Exposure-response analyses for the efficacy parameter urinary albumin:creatinine ratio and safety markers eGFR and serum potassium quantify effects on different time-scales and indicate doses of finerenone 10 and $20 \mathrm{mg}$ once daily as safe and efficacious at reducing albuminuria for pivotal studies. 


\section{Introduction}

Aldosterone is a steroid hormone that, apart from its renal effects on sodium retention and potassium excretion, exerts important pro-inflammatory/-fibrotic effects contributing to the pathogenesis of cardiorenal diseases. The current steroidal aldosterone blockers (i.e., mineralocorticoid receptor [MR] antagonists [MRAs]) spironolactone and eplerenone have a class I recommendation for patients with chronic heart failure ( $\mathrm{CHF}$ ) and a reduced left ventricular function (heart failure with reduced ejection fraction $[\mathrm{HFrEF}])$. However, their use is limited due to the potential development of hyperkalemia (i.e., an increase of serum potassium above $5.5 \mathrm{mmol} / \mathrm{L}$ ) and kidney dysfunction, particularly when given on top of standard of care such as angiotensin converting enzyme inhibitors (ACEIs) or angiotensin receptor blockers (ARBs) among patients with concomitant renal insufficiency. This intrinsic risk is even more relevant for patients with chronic kidney disease (CKD). On the other hand, small exploratory clinical studies demonstrated significant reduction of the most important pharmacodynamic parameter albuminuria/ proteinuria in these patients. In fact, addition of spironolactone to ACEIs and/or ARBs was found to increase the risk of hyperkalemia three-fold in patients with CKD, as deduced from a comprehensive meta-analysis [1].

Finerenone is a novel, potent, non-steroidal, selective MRA being developed for the treatment of patients with type 2 diabetes (T2D) and CKD. Compared with currently available steroid-based MRAs, it has greater MRA-receptor selectivity than spironolactone and higher receptor affinity than eplerenone in vitro [2]. Favorable properties were shown in preclinical studies [3], and clinically in ARTS (minerAlocorticoid Receptor antagonist Tolerability Study), where finerenone doses of $2.5-10 \mathrm{mg} /$ day were shown to lead to significantly smaller mean increases in serum potassium than spironolactone (mean dose $37 \mathrm{mg}$ / day) in patients with CHF and CKD stage III. As an exploratory outcome, a reducing effect on albuminuria from baseline was observed with finerenone compared with placebo [4]. The efficacy and safety were further investigated in ARTS-DN (ARTS-Diabetic Nephropathy), in which different once-daily (OD) oral doses of finerenone and placebo were given to patients with T2D and persistent albuminuria (urinary albumin:creatinine ratio $[\mathrm{UACR}] \geq 30 \mathrm{mg} / \mathrm{g}$ ) receiving a renin-angiotensin system (RAS) blocker; the addition of finerenone resulted in a dose-dependent reduction in UACR that was statistically significant compared with placebo for the 7.5-20 mg OD doses [5]. Similar results were obtained in the Japanese study (ARTS-DN Japan), in which finerenone again reduced albuminuria, without adverse effects on serum potassium levels or renal function, in Japanese patients with T2D and CKD [6]. The safety, tolerability, and pharmacokinetics of finerenone were evaluated in healthy male volunteers in two randomized, single-center studies spanning a dose range of $1-80 \mathrm{mg}$ showing dose-linear pharmacokinetics with rapid absorption and elimination [7]. In a renal impairment study, exposure to finerenone was not affected by mild renal impairment, whereas moderate or severe renal impairment increased integral exposure with moderate-to-high inter-individual variability, without a consistent effect on maximum concentration $\left(C_{\max }\right)$. Renal elimination of finerenone is minimal (less than $1 \%$ of dose). However, changes in exposure may occur because of the effects of renal impairment on non-renal routes of elimination [8]. In a hepatic impairment study, finerenone area under the plasma concentration-time curve (AUC) and unbound finerenone AUC were $38 \%$ and 55\% greater, respectively, in participants with moderate hepatic impairment than in healthy participants, whereas $C_{\max }$ was unchanged. No clear effects on AUC or $C_{\max }$ were seen in participants with mild hepatic impairment [9]. Crossover studies assessed the mass balance, absolute bioavailability, and effects of erythromycin, verapamil, and gemfibrozil on the systemic exposure of finerenone, complemented by in vitro studies $[10,11]$.

The phase I studies with pharmacokinetic components did not include patients with CKD, and thus no relevant pharmacodynamics were expected. Although providing initial evidence of safety and efficacy, ARTS was not focused on T2D with CKD. The aim of the present study was to use the sparse data collected in the phase IIb ARTS-DN and ARTS-DN Japan studies and characterize pharmacokinetic variability, investigate covariate effects, and discern the pharmacokinetic/pharmacodynamic (PK/ PD) relationship to relevant safety (serum potassium and estimated glomerular filtration rate [eGFR]) and efficacy parameters (UACR) in adult patients with T2D and CKD. Increasing albuminuria as measured by UACR is a robust predictor of renal and cardiovascular risk, and retrospective and observational studies have demonstrated a strong association between changes in albuminuria and risk of end-stage renal disease (ESRD) and mortality [12-15]. A population pharmacokinetic (popPK) model utilized data from all subjects of the phase IIb ARTS-DN and ARTS-DN Japan studies simultaneously to characterize the concentration-time course of the drug for both the study population and individual subjects [16]. The developed model was further applied to simulate the dose-exposure-response relationships for relevant safety and pharmacodynamic parameters in a T2D with CKD study population as a phase III scenario. 


\section{Methods}

PopPK/pharmacodynamic (PopPK/PD) models for finerenone were developed continuously alongside its clinical development (Table 1). The models identified, based on clinical phase I and IIa data, served as a starting point for the current analysis. Relevant information on the model development history is presented in Electronic Supplementary Material (ESM) Phase IIa Models.

The current analysis focuses on data from the two phase IIb studies, ARTS-DN (NCT01874431) and ARTS-DN Japan (NCT01968668). ARTS-DN was a global study that did not include patients from Japan, who were investigated in the dedicated study ARTS-DN Japan. Dose-exposure-response analyses were first conducted on ARTSDN data alone, and then on a combined ARTS-DN and ARTS-DN Japan dataset termed ARTS-DN ${ }^{+J P}$ hereafter. Further details on the studies, including analytical methods, are summarized in the ESM Methods and have been published in more detail previously $[5,6,17]$. Tables 2 and 3 provide summary characteristics and statistics of the studies relevant for the analysis presented here. The primary efficacy endpoint in these studies was effect of treatment on UACR after 90 days compared with baseline; the main safety markers were change in serum potassium concentration and eGFR.

For the current analysis, the phase IIa pharmacokinetic and PK/PD models for the aforementioned markers (ESM Phase IIa Models) were updated by parameter estimation first with ARTS-DN data, and then using the ARTS- $\mathrm{DN}^{+\mathrm{JP}}$ dataset (Tables 2 and 3) and, if needed, the structural models were re-evaluated. Additional information on model development, simulation specifications, and computational tools used are presented in the ESM Methods. Confidence intervals (CIs) throughout the text correspond to $90 \%$ CIs. For simulations, a hypothetical phase III scenario has been considered with population characteristics as defined in Table 4.

Notably, two different methods were used to derive eGFR. The eGFR derived using the Modification of Diet in Renal Disease (MDRD) study equation (here termed eGFR-MDRD) is historically the more widely used biomarker [18-20], whereas eGFR according to the CKD Epidemiology Collaboration (CKD-EPI) equation (here termed eGFR-EPI) is calculated using a more recent formula more specific for CKD [21, 22] (see also Eqs. S9 and S10 in the ESM Functional Forms). The ARTS-DN studies evaluated eGFR-EPI and this was used in the exposure-response analysis presented here. For consistency and comparability with historical finerenone studies analyzed with popPK models, eGFR-MDRD was used as a covariate for pharmacokinetics in the popPK analysis presented here.

Also of note, in the current analysis serum potassium was fitted and simulated and population fractions crossing thresholds of 5.5 and $6.0 \mathrm{mmol} / \mathrm{L}$ were derived irrespective of study continuation, as further detailed in the ESM Methods. This does not directly correspond to the prespecified secondary outcome of hyperkalemia in ARTSDN that included study discontinuation [5].

\section{Results}

\subsection{Pharmacokinetics}

The phase IIa two-compartmental pharmacokinetic model (ESM Phase IIa Models) with absorption through a series

Table 1 Model overview

\begin{tabular}{|c|c|c|c|}
\hline Models & Variable & Data included in the analysis & Notes \\
\hline Phase I models & PKs & Phase I & $\begin{array}{l}\text { Starting point for phase IIa model } \\
\text { development } \\
\text { Not reported }\end{array}$ \\
\hline Phase IIa models (ARTS) & $\begin{array}{l}\text { PKs, UACR, serum potassium, } \\
\text { and eGFR-MDRD }\end{array}$ & ARTS & $\begin{array}{l}\text { Starting point for phase IIb model } \\
\text { development } \\
\text { Models described in ESM Phase IIa } \\
\text { models } \\
\text { Parameters of the PK and UACR } \\
\text { models reported in ESM Tables } \\
\text { S1 and S2 }\end{array}$ \\
\hline ARTS-DN models & $\begin{array}{l}\text { PKs, UACR, serum potassium, } \\
\text { and eGFR-EPI }\end{array}$ & ARTS-DN & Current analysis \\
\hline $\begin{array}{l}\text { ARTS-DN }^{+J P} \text { models (final } \\
\text { models) }\end{array}$ & $\begin{array}{l}\text { PKs, UACR, serum potassium, } \\
\text { and eGFR-EPI }\end{array}$ & ARTS-DN and ARTS-DN Japan & Current analysis \\
\hline
\end{tabular}

$e G F R-E P I$ estimated glomerular filtration rate according to the Chronic Kidney Disease Epidemiology Collaboration equation, $e G F R-M D R D$ estimated glomerular filtration rate according to the Modification of Diet in Renal Disease study equation, ESM Electronic Supplementary Material, $P K s$ pharmacokinetics, UACR urinary albumin:creatinine ratio 
Table 2 Summary statistics on the datasets ARTS-DN and ARTS-DN Japan on subjects and observations

\begin{tabular}{llll}
\hline Statistics & ARTS-DN & ARTS-DN Japan & ARTS-DN $^{+J P}$ \\
\hline Subjects randomized $(n)$ & 823 & 96 & 919 \\
Subjects in PK/PD analysis dataset $(n)$ & 799 & 94 & 893 \\
Placebo & 94 & 12 & 106 \\
Finerenone 1.25 mg OD & 94 & 12 & 106 \\
Finerenone 2.5 mg OD & 90 & 12 & 102 \\
Finerenone 5.0 mg OD & 97 & 12 & 109 \\
Finerenone 7.5 mg OD & 96 & 12 & 108 \\
Finerenone 10 mg OD & 93 & 11 & 104 \\
Finerenone 15 mg OD & 121 & 11 & 132 \\
Finerenone 20 mg OD & 114 & 12 & 126 \\
UACR observations ( $n$ ) & 5099 & 567 & 5666 \\
Serum potassium concentration observations $(n)$ & 6014 & 954 & 6968 \\
eGFR-EPI observations $(n)$ & 5925 & 947 & 6872 \\
Ethnicity in PK/PD dataset (\%) & & & 75.7 \\
Caucasians & 84.6 & 0.0 & 9.1 \\
Asians & 10.1 & 0.0 & 10.5 \\
Japanese & 0.0 & 100 & 2.9 \\
African Americans & 3.3 & 0.0 & 1.8 \\
Other/not reported & 2.0 & 0.0 & 787 \\
Subjects in PK dataset $(n)$ & 705 & 82 & 4597 \\
PK observations above LLOQ $(n)$ & 4109 & 488 & \\
PK observations below LLOQ (of all non-missing & $544(11.3)$ & $63(11.1)$ & \\
observations) [ $n$ (\%)] & & & \\
\hline
\end{tabular}

Subjects without any valid pharmacokinetic observations or with implausible observations only were excluded from the analysis. Statistics are also included for the combined ARTS-DN and ARTS-DN Japan dataset, defined as ARTS-DN ${ }^{+\mathrm{JP}}$

$e G F R-E P I$ estimated glomerular filtration rate according to the Chronic Kidney Disease Epidemiology Collaboration equation, $L L O Q$ lower limit of quantification, $O D$ once daily, $P D$ pharmacodynamic, $P K$ pharmacokinetic, $U A C R$ urinary albumin:creatinine ratio

Table 3 Summary statistics of selected covariates and response parameters determined at baseline from all subjects used for pharmacokinetic/ pharmacodynamic analysis, for ARTS-DN and ARTS-DN Japan

\begin{tabular}{|c|c|c|c|c|c|c|}
\hline \multirow[t]{3}{*}{ Statistic } & \multicolumn{6}{|c|}{ Variable baseline percentiles } \\
\hline & \multicolumn{2}{|c|}{ 5th percentile } & \multicolumn{2}{|c|}{ 50th percentile } & \multicolumn{2}{|c|}{ 95th percentile } \\
\hline & ARTS-DN & $\begin{array}{l}\text { ARTS-DN } \\
\text { Japan }\end{array}$ & ARTS-DN & ARTS-DN Japan & ARTS-DN & ARTS-DN Japan \\
\hline Body weight (kg) & 64.1 & 54 & 90.6 & 71.6 & 126.3 & 100 \\
\hline BMI $\left(\mathrm{kg} / \mathrm{m}^{2}\right)$ & 24.2 & 21.1 & 31.1 & 26.5 & 41.7 & 34.6 \\
\hline Age (years) & 49 & 44 & 65 & 64 & 78 & 78 \\
\hline eGFR-MDRD (mL/min/1.73 m²) & 33.5 & 41.0 & 63.9 & 61.5 & 102.5 & 87.2 \\
\hline eGFR-EPI (mL/min/1.73 m²) & 33.3 & 42.3 & 66.3 & 64.6 & 101.3 & 85.2 \\
\hline Serum potassium concentration $(\mathrm{mmol} / \mathrm{L})$ & 3.6 & 3.6 & 4.3 & 4.2 & 5.0 & 4.8 \\
\hline UACR (combined) (g/kg) & 33.7 & 38.5 & 192.4 & 216.4 & 1626 & 1345 \\
\hline
\end{tabular}

$B M I$ body mass index, eGFR-EPI estimated glomerular filtration rate according to the Chronic Kidney Disease Epidemiology Collaboration equation, $e G F R-M D R D$ estimated glomerular filtration rate according to the Modification of Diet in Renal Disease study equation, UACR urinary albumin:creatinine ratio 
Table 4 Hypothetical phase III simulation scenario

\begin{tabular}{ll}
\hline Inclusion criteria & Serum potassium concentration $<4.8 \mathrm{mmol} / \mathrm{L}$ at run-in and screening. No reassessment allowed \\
& UACR $\geq 30 \mathrm{mg} / \mathrm{g}$ in two of three morning samples at run-in and screening \\
& $25 \leq \mathrm{eGFR} \leq 90 \mathrm{~mL} / \mathrm{min} / 1.73 \mathrm{~m}^{2}$ at run-in and screening \\
Exclusion criteria & $\mathrm{UACR}>3500 \mathrm{mg} / \mathrm{g}$ in any of the samples at run-in or screening \\
Study design & OD administration and full compliance during a treatment period of 180 days \\
& Five visits during treatment at which finerenone concentration, UACR, serum potassium con- \\
& centration, and eGFR are measured: days $1,30,60,90$, and 180
\end{tabular}

$e G F R$ estimated glomerular filtration rate, $O D$ once-daily, $U A C R$ urinary albumin:creatinine ratio

of transit compartments, together with a fixed lag time, and first-order elimination could adequately describe the finerenone concentration-time profiles over the 1.25-20 mg OD dose range in ARTS-DN. The updated parameter estimates in comparison with the phase IIa model parameter estimates can be found in ESM Table S1. The significant covariate effects for body weight (BW) and eGFR-MDRD from the prior phase IIa pharmacokinetic model were tested and retained in the updated model. The pharmacokinetics were adequately described by the model because observed and predicted median and variability of the concentration-time profile closely resemble each other (see ESM Figure S1). The pharmacokinetics were dose- and time-linear over the investigated dose range and treatment period. Taking differences in the distribution of BW and eGFR-MDRD into account, the pharmacokinetics of finerenone were comparable between phase I, IIa, and IIb study populations. The apparent volume of distribution of the central compartment $\left(\mathrm{V}_{\mathrm{c}} / F\right.$, and as a consequence apparent volume of distribution $[\mathrm{V} / F]$ ) increased with $\mathrm{BW}$ and apparent clearance $(\mathrm{CL} / F)$ decreased with decreasing eGFR-MDRD. In addition, the oral bioavailability $(F)$ increased with decreasing eGFR-MDRD (ESM Table S1). Therefore, both effects led to an increase in exposure with decreasing eGFR-MDRD. The covariates had a significant impact on pharmacokinetic model parameters based on the minimum value of the objective function (MVOF) and, for eGFR-MDRD, on the AUC at steady state $\left(\mathrm{AUC}_{\mathrm{ss}}\right)$. A subject with eGFR-MDRD of $30 \mathrm{~mL} / \mathrm{min} / 1.73 \mathrm{~m}^{2}$ had a $32.9 \%$ higher $\mathrm{AUC}_{\mathrm{ss}}$ and a $17.5 \%$ higher $C_{\max }$ than a subject with eGFR-MDRD of $90 \mathrm{~mL} / \mathrm{min} / 1.73 \mathrm{~m}^{2}$. The $C_{\max }$ of a subject with a BW of $50 \mathrm{~kg}$ was $43.1 \%$ higher than that of a subject of BW $100 \mathrm{~kg}$. After the second dose of finerenone, i.e., after 2 days' dosing, full (99\%) steady-state conditions were reached with regards to pharmacokinetics.

Dose-normalized $\mathrm{AUC}_{\mathrm{ss}}$ and $C_{\mathrm{max}}$ at steady state $\left(C_{\mathrm{max}, \mathrm{ss}}\right)$, as calculated from empirical Bayes estimates, were $0.4 \%$ and $7.4 \%$ higher, respectively, in the Japanese population than in the global population (Fig. 1). No change in the structural model (i.e., ethnicity effect) was necessary to describe the pharmacokinetics after adding data from Japanese patients. The model parameters were updated, resulting in small changes compared with the parameters from the model based on global data only (ESM Table S1). Baseline eGFRMDRD was $3.5 \%$ lower in the Japanese population than the global population, resulting in a very minor difference in $\mathrm{AUC}_{\mathrm{ss}}(0.4 \%)$. BW was $20.5 \%$ lower in the Japanese study population than in the global population. This translates into a $9 \%$ lower apparent total volume and a somewhat higher $C_{\text {max,ss }}(7.4 \%)$ and lower trough concentration at steady state in Japanese patients than in the global population.

\subsection{Pharmacokinetics and Pharmacodynamics}

\subsubsection{Urinary Albumin:Creatinine Ratio}

UACR at baseline was higher in the phase IIb population than in the phase IIa study population, reflecting that increased albuminuria was an inclusion requirement for ARTS-DN but not for ARTS. Consequently, the UACR levels were under-predicted by the unchanged phase IIa UACR model. After parameter re-estimation and estimation of separate UACR baseline values for patients with very high albuminuria (UACR at screening $>300 \mathrm{mg} / \mathrm{g}$ ), the UACR data were adequately described because the median of the observations falls within the uncertainty of the model-predicted median (Fig. 2 and ESM Figures S2 and S3). The percentages of subjects who reached predefined target UACRs (UACR at day 90 [UACR_day ${ }_{90}$ ]/ UACR at baseline [UACR_baseline] $\leq 0.5, \leq 0.6, \leq 0.7$, or $\leq 0.8$ ) were slightly over-predicted. This was accepted because observations were within the CI (ESM Figure $\mathrm{S} 4$, left panels). UACR decreased with increasing finerenone plasma exposure and, consequently, the predicted percentage of subjects reaching a target UACR increased with dose. The drug effect was proportional to the baseline UACR. Because the ratio is calculated as UACR_day ${ }_{90} /$ UACR_baseline, the drug effect on the ratio is independent of the baseline UACR and is the same for normal, high, and very high albuminuria. A time delay was observed between the increase in finerenone concentration and the decrease in UACR. The model-predicted time to reach $99 \%$ of the steady-state drug effect of finerenone on UACR was 138 days. 


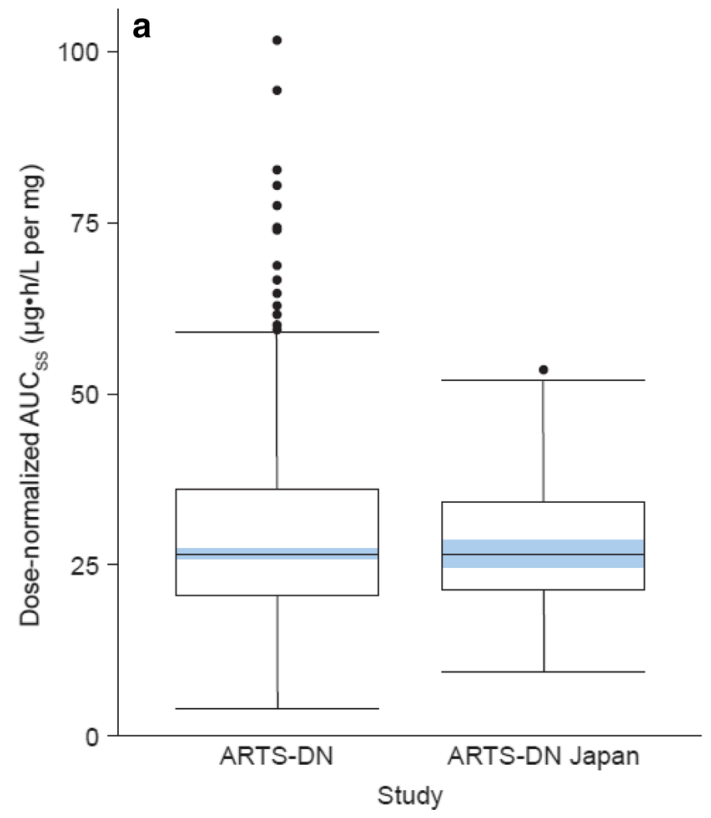

Fig. 1 Box plot of dose-normalized a $\mathrm{AUC}_{\mathrm{ss}}$ at steady state and $\mathbf{b}$ $C_{\text {max,ss }}$ (day 30) of once-daily administration for the ARTS-DN and ARTS-DN Japan populations. The median dose-normalized AUC ss and $C_{\text {max,ss }}$ values were 26.6 and $26.7 \mu \mathrm{g} \cdot \mathrm{h} / \mathrm{L} / \mathrm{mg}$ and 6.8 and

All parameters could be estimated with good precision because all relative standard errors (RSEs) were $<50 \%$ (ESM Table S2). Power and log-linear concentration-effect relationships were statistically preferred over the maximum inhibition $\left(I_{\max }\right)$ model because the MVOFs were significantly lower for the former. However, these models were not suitable for extrapolation to higher doses because, given the model parameter estimates, UACR would become negative at doses $>20 \mathrm{mg}$. Therefore, an $I_{\max }$ relationship was preferred over the two more descriptive alternatives.

There were no apparent differences between Japanese and non-Japanese subjects in the PK/PD relationship for UACR. The predicted percentage of patients who reached the target UACRs were generally lower in the Japanese than in the global study, but the CIs of the median predicted percentages of the two populations overlapped (ESM Figure S4, right panel). Furthermore, the CIs of the median predicted percentages of subjects for the Japanese study were wider than those for the non-Japanese study, because of the smaller number of subjects in the Japanese study.

\subsubsection{Serum Potassium Concentration}

The serum potassium concentrations in the phase IIb studies were predicted adequately by the phase IIa model with a linear concentration-effect relationship for doses up to finerenone $15 \mathrm{mg}$ OD. For the $20 \mathrm{mg}$ OD dose, the

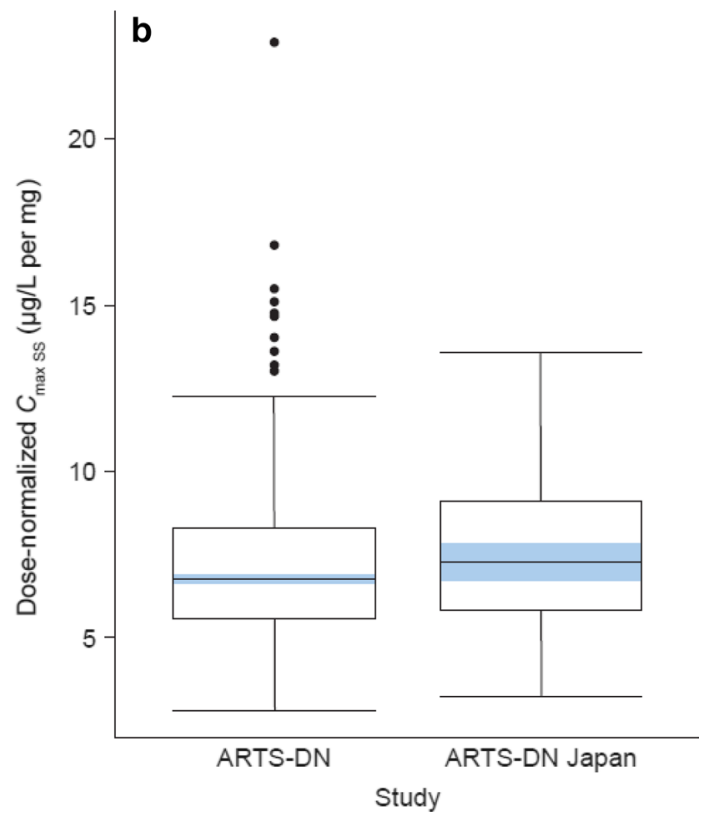

$7.3 \mu \mathrm{g} / \mathrm{L} / \mathrm{mg}$, for the ARTS-DN and ARTS-DN Japan studies, respectively. $A U C_{s s}$ area under the plasma concentration-time curve at steady state, $C_{\max S S}$ maximum concentration at steady state

increase in serum potassium concentration was overpredicted; therefore, alternative models were evaluated. The concentration-effect relationship for serum potassium was well-described by a log-linear model indicating attenuation of effect gains at high exposures (Fig. 2 and ESM Figures S2 and S5). The finerenone drug effect was proportional to the baseline serum potassium concentration, indicating a larger absolute effect for subjects with a higher baseline serum potassium concentration. The delayed increase in serum potassium concentration was described using a turnover model. The serum potassium concentration increased with increasing finerenone plasma exposure and, consequently, the predicted percentage of subjects with one or more serum potassium values $>5.5$ or $>6.0 \mathrm{mmol} / \mathrm{L}$ at any visit increased with dose (ESM Figure S6). The proportion of subjects crossing these thresholds was dependent on the baseline serum potassium concentration, i.e., subjects with a higher baseline serum potassium concentration are more likely to cross the thresholds. All parameters could be estimated with good precision (RSEs $<50 \%$; ESM Table S3). The model predicted that $99 \%$ of the steady-state drug effect was reached after 20 days.

There were no apparent differences between Japanese and non-Japanese patients in the PK/PD relationship for serum potassium concentration. With respect to the structural model parameters, serum potassium baseline was determined to be significantly lower $(3.1 \%$; $95 \% \mathrm{CI}$ 
Fig. 2 Predicted and observed a UACR, b absolute serum potassium concentration, and $\mathbf{c}$ relative change from baseline in eGFR-EPI versus finerenone $\mathrm{AUC}_{\mathrm{ss}}$. The model was fitted to individual data. For plotting purposes, the data were binned in 20 categories based on equal numbers of records. Blue dashed lines: reference/threshold lines; dark gray dashed lines: simulated $\mathrm{AUC}_{\mathrm{ss}}$ for a typical subject for doses of 10, 20, and $30 \mathrm{mg}$; black solid lines: 5th and 95th percentiles of the observations; black dots: medians of the observations; red solid line and dashed lines: median predictions and 5th and 95th percentiles of the predictions; gray areas: $90 \%$ confidence intervals of the median and 5th and 95th percentiles. $A U C_{s s}$ area under the plasma concentration-time curve at steady state, eGFR-EPI estimated glomerular filtration rate according to the Chronic Kidney Disease Epidemiology Collaboration equation, UACR urinary albumin:creatinine ratio

\section{8-4.5) in ARTS-DN Japan than in ARTS-DN (ESM}

Table S3). No significant differences were found for other structural parameters. The predicted percentage of subjects who reached one or more serum potassium concentrations $>5.5 \mathrm{mmol} / \mathrm{L}$ during the study period was lower in the Japanese study than in the global study (ESM Figure S6).

\subsubsection{Estimated Glomerular Filtration Rate}

The eGFR-EPI values were predicted adequately by the phase IIa eGFR-MDRD model for doses up to finerenone $10 \mathrm{mg}$ OD as the observed and predicted median and variability in the change in eGFR-EPI closely resemble each other. However, the effect on eGFR-EPI was over-predicted for the higher doses. A power concentration-effect relationship was shown to be significantly better $(p<0.001)$ than the original linear relationship that was determined in phase IIa. It was also superior to log-linear and (sigmoid) $I_{\max }$ relationships. The concentration-effect relationship for eGFR-EPI was well-described by the power model, as can been seen in the plot of the comparison of the predicted and observed eGFR-EPI concentrations versus $\mathrm{AUC}_{\mathrm{ss}}$ (Fig. 2 and ESM Figures S2 and S7) and in the plot of the comparison of the predicted and observed percentages of subjects with a decrease in eGFR-EPI thresholds (ESM Figure S8, left panels). eGFR decreases with increasing finerenone plasma concentration and, consequently, the predicted percentage of subjects with a decrease in eGFR-EPI thresholds $(\geq 30 \%, \geq 40 \%$, and $\geq 57 \%)$ at day 90 increases with dose. All parameters could be estimated with good precision (RSEs $<50 \%$; ESM Table S4). A time delay was observed between the increase in finerenone concentration and the decrease in eGFR-EPI; the time to reach 99\% steady-state drug effect was 85 days.

There were no apparent differences between Japanese and non-Japanese subjects in the PK/PD relationship for eGFREPI. The model-based analysis indicated that the percentage of patients reaching $\geq 30 \%$ decrease in eGFR-EPI was lower
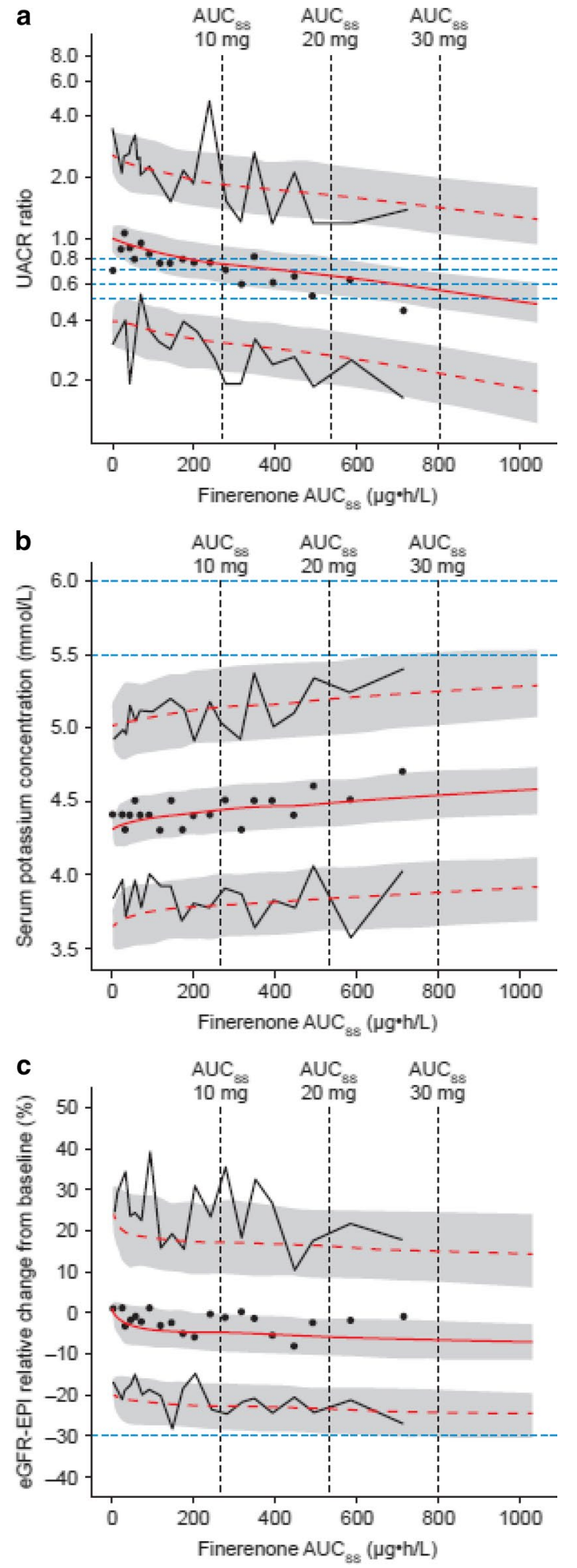

in the Japanese than in the global study at the same dose level and treatment period, but the CIs overlapped (ESM Figure S8, right panels). 


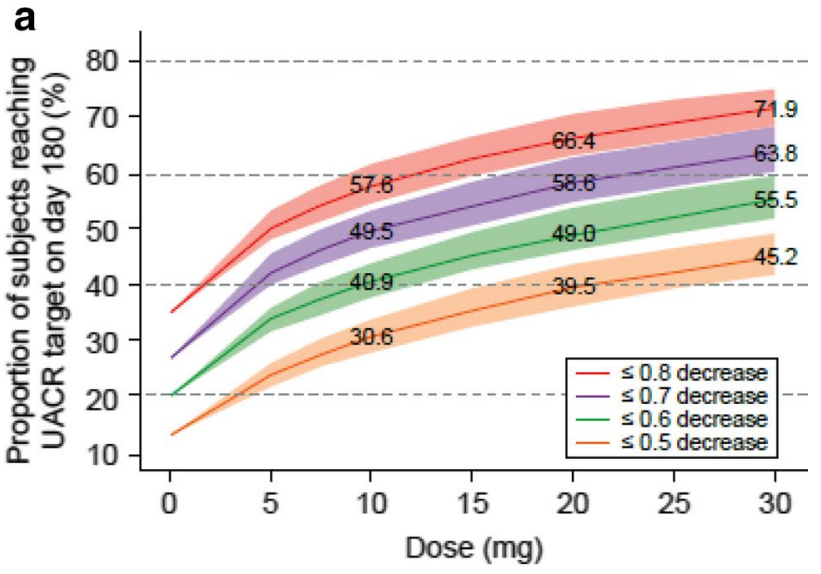

b
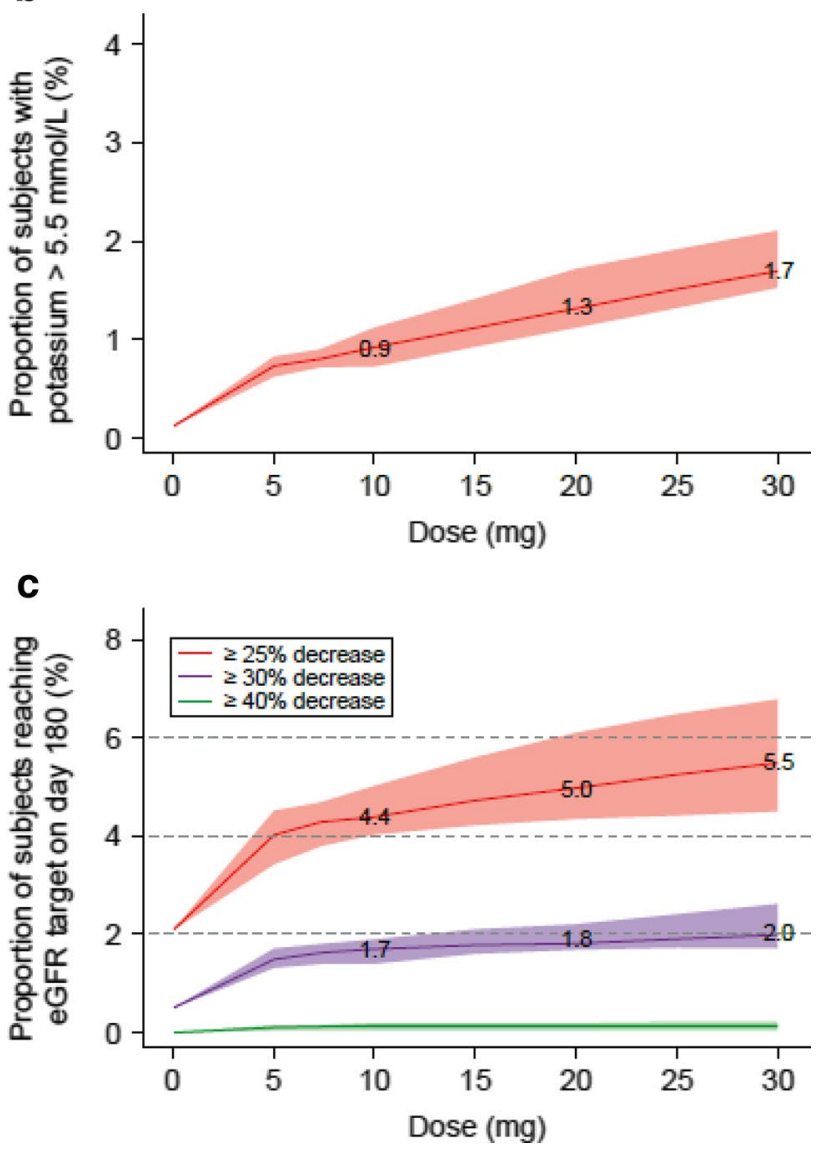

Fig. 3 Simulated dose-response curves for a UACR, b serum potassium concentration, and $\mathbf{c}$ eGFR-EPI for a phase III scenario (Table 3) following once-daily administration of placebo $(0 \mathrm{mg})$ and different doses of finerenone $(5.0,7.5,10,15,20$, and $30 \mathrm{mg})$. Presented are the median predicted percentages of subjects (solid lines) with $90 \%$ confidence intervals (shaded areas), and the percentages of subjects reaching the thresholds following finerenone 10,20 , and $30 \mathrm{mg}$ once daily. a Percentage of subjects who reach specified UACR targets at day 180 . b Percentage of subjects who have one or more serum potassium concentration value $>5.5 \mathrm{mmol} / \mathrm{L}$ at any visit during treatment. $\mathbf{c}$ Percentage of subjects who have specified decreases from baseline in eGFR at day 180. eGFR estimated glomerular filtration rate, eGFR-EPI estimated glomerular filtration rate according to the Chronic Kidney Disease Epidemiology Collaboration equation, UACR urinary albumin:creatinine ratio

\subsection{Simulations}

Simulations shown in Fig. 3 consider doses up to finerenone $30 \mathrm{mg}$ OD and effects after 180 days, thus extrapolating for higher doses than the investigated $20 \mathrm{mg}$ OD and for longer times than the investigated 90 days in ARTS-DN, given that the model predicts that steady-state pharmacodynamic effects (for UACR) are only achieved beyond 90 days. In the following sections, the differences for the exemplary extrapolated high dose of $30 \mathrm{mg}$ OD will be further described.

\subsubsection{Urinary Albumin:Creatinine Ratio}

Simulations indicated that the percentage of subjects reaching target UACRs at day 180 increased with finerenone dose. At a dose of $20 \mathrm{mg} \mathrm{OD}$, the proportion of subjects reaching a target ratio UACR at day 180 of $\leq 0.8, \leq 0.7, \leq 0.6$, and $\leq 0.5$ is $66.4 \%, 58.6 \%, 49.0 \%$, and $39.5 \%$, respectively. Increasing the dose from $20 \mathrm{mg}$ OD to $30 \mathrm{mg}$ OD is expected to result in a respective increase of 5.5\%, 5.2\%, 6.5\%, and 5.7\% (Fig. 3).

\subsubsection{Serum Potassium Concentration}

Simulations indicated that the percentage of subjects in the ARTS-DN population having one or more serum potassium values $>5.5 \mathrm{mmol} / \mathrm{L}$ at any visit during a 180-day treatment period increases from $1.3 \%$ (90\% CI 1.1-1.7) to $1.7 \%$ (90\% CI 1.5-2.1) when increasing the dose from $20 \mathrm{mg}$ OD to $30 \mathrm{mg}$ OD (Fig. 3). No subjects are expected to have one or more potassium values above $6.0 \mathrm{mmol} / \mathrm{L}$.

\subsubsection{Estimated Glomerular Filtration Rate}

Simulations showed that when the finerenone dose is increased from $20 \mathrm{mg}$ OD to $30 \mathrm{mg}$ OD, the percentage of subjects with a decrease (change from baseline) in eGFREPI at day 180 of $\geq 25 \%$ and $\geq 30 \%$ goes up from $5.0 \%$ (90\% CI 4.3-6.1) to 5.5\% (90\% CI 4.5-6.8) and from 1.8\% (90\% CI 1.6-2.2) to $2.0 \%$ (90\% CI 1.7-2.6), respectively. Fewer than $0.1 \%$ of subjects are predicted to have a decrease in eGFR-EPI of $\geq 40 \%$ and $\geq 57 \%$, respectively, at day 180 following OD administration of finerenone 20 and $30 \mathrm{mg}$ (Fig. 3).

\section{Discussion}

Population pharmacokinetic and PK/PD models were developed to characterize the pharmacokinetics of finerenone and its effect on UACR, serum potassium concentration, and eGFR in patients with T2D and CKD using data from the phase IIb ARTS-DN global and Japan studies, including a comparison of the two studies. The developed models were 
further used to simulate the dose-exposure-response relationships for the biomarkers in a study population with T2D and CKD as a phase III scenario.

Methods based on predefined event counting generally consider the data at one timepoint, visit, or dose group, whereas continuous PK/PD models take the totality of the data into account. For this reason, PK/PD models can be considered more robust than predefined event counting methods.

\subsection{Pharmacokinetics}

Generally, the pharmacokinetics of finerenone are wellunderstood and were described adequately in the ARTSDN population by a two-compartmental pharmacokinetic model with absorption through a series of transit compartments, together with a fixed lag time, and first-order elimination following administration of finerenone at dose levels of 1.25-20 mg OD. The central and peripheral volumes of distribution were assumed to be equal to avoid over-parameterization. This may limit the possibility to identify covariate effects on the distribution. In addition, the terminal elimination after $24 \mathrm{~h}$ may not be adequately predicted. $\mathrm{AUC}_{\mathrm{ss}}$ and $C_{\max }$ are likely not influenced by this model reduction. The covariates eGFR-MDRD and BW were found to influence the pharmacokinetics of finerenone. The pharmacokinetics of finerenone in the ARTS-DN population is comparable with the pharmacokinetics observed in the phase IIa and phase I populations, taking the differences in eGFR-MDRD and BW into account.

The pharmacokinetics of finerenone were dose-linear in previous studies across the investigated dose range of $1-80 \mathrm{mg}$ [7]. The current analysis confirms dose-linearity in the clinically relevant dose range investigated in ARTS-DN. The current analysis also indicates the pharmacokinetics to be linear over time and steady state to be reached within 2 days.

\subsection{Pharmacology}

The pharmacology of MR antagonism is diverse. The renal epithelial sodium/potassium transport controlled by MR is the result of a complex interplay of transcriptionally induced target gene products (such as ion channels) leading to sodium retention and potassium release within hours after treatment initiation. A potential MRA-induced accumulation of serum potassium among kidney disease patients already under RAS blockade might develop within days, while a decrease in UACR is the result of even more complex antiinflammatory/-fibrotic long-term effects mediated by MR antagonism. Finerenone is distinguishable from steroidal MRAs based on differential MR nuclear co-factor binding yielding a relatively more pronounced anti-fibrotic effect for a given effect on renal sodium/potassium homeostasis than in steroidal MRAs [3, 23].

The effect of finerenone on the efficacy marker UACR was adequately captured by a turnover model with an inhibiting effect on $k_{\text {in }}$ (zero-order production rate constant). UACR decreases with increasing finerenone concentrations. The maximum effect model indicates saturation at high exposures. Separate UACR baseline values were estimated for patients with very high albuminuria (UACR at screening $>300 \mathrm{mg} / \mathrm{g}$ ) and patients with UACR at screening $\leq 300 \mathrm{mg} / \mathrm{g}$. The potency estimate was the same for patients with normal, high, and very high albuminuria. For a dose of $20 \mathrm{mg}$, the model predicted $66.1 \%$ (90\% CI $58.9-73.2$ ), $58.0 \%$ (90\% CI 50.0-65.2), $48.2 \%$ (90\% CI $41.1-55.4$ ), and $36.6 \%$ (90\% CI $29.5-44.6$ ) of subjects to have $\mathrm{UACR} \leq 0.8, \leq 0.7, \leq 0.6$, and $\leq 0.5$ at day 90 in ARTSDN (ESM Figure S6). Published studies indicate an almost linear, non-saturating relationship between albuminuria and renal risk [24] and between albuminuria and cardiovascular and heart failure risk [25]. Also, increasing albuminuria as measured by UACR is a robust predictor of renal and cardiovascular risk, and retrospective and observational studies have demonstrated a strong association between changes in albuminuria and risk of ESRD and mortality [12, 13].

For the safety marker serum potassium concentration, the concentration-effect relationship was adequately described by a log-linear model. The model predicted an increase in serum potassium concentration with increasing finerenone concentration. Of the subjects in ARTS-DN, 7.9\% (90\% CI 3.5-15.0) and $0.0 \%$ (90\% CI 0-1.8) were predicted to have one or more serum potassium values $\geq 5.5$ or $\geq 6.0 \mathrm{mmol} / \mathrm{L}$, respectively, following OD administration of finerenone $20 \mathrm{mg}$ (ESM Figure S6). This percentage was found to be dependent on the baseline serum potassium concentration [5]. Generally, the percentages of subjects above serum potassium thresholds derived in the current analysis are higher than the numbers previously reported based on statistical analysis. The latter are not only based on single elevated potassium findings but also on subsequent discontinuation of study drug, which was generally only considered upon reassessment confirming elevated serum potassium, e.g., for the $20 \mathrm{mg}$ group this was found for $1.7 \%$ of subjects [5].

The effect of finerenone on eGFR-EPI was adequately described by a power model for the entire dose range describing a sustained decrease with increasing finerenone concentrations during active treatment, although the effect was small considering overall variability [5]. An initial eGFR decline with the introduction of RAS-blocking therapy is postulated to represent a reduction in intra-glomerular pressure rather than intrinsic renal damage, and this initial reduction may serve as a predictor of future therapeutic benefit including long-term reno-protection and preservation of GFR [26, 27]. For a dose of $20 \mathrm{mg}$ OD, $1.8 \%$ (90\% CI 
0-4.5), 0\% (90\% CI 0-0.9), and 0\% (90\% CI 0-0) of subjects in ARTS-DN were predicted to have a decrease in eGFR of $\geq 30 \%, \geq 40 \%$, and $\geq 57 \%$ at day 90 , respectively. Overall, the effect was rather minor; additionally, the primary analysis found no differences in the incidence of the prespecified secondary outcome of an eGFR decrease of $30 \%$ or more between the placebo and finerenone groups [5].

\subsection{Simulations}

Simulations were performed to predict the effect of finerenone on UACR, serum potassium concentration, and eGFR-EPI in a phase III scenario (Table 4). As the developed pharmacokinetic and pharmacodynamic models do not capture disease progression, the simulations were limited to 180 days, which is a timeframe in which no, or limited, disease progression is expected. The effect of finerenone on the efficacy and safety markers was simulated for placebo and OD doses of $5,7.5,10,15,20$, and $30 \mathrm{mg}$, the last being an extrapolation beyond the investigated dose range. However, the expected median exposure of approximately $800 \mu \mathrm{g} \cdot \mathrm{h} / \mathrm{L}$ for a dose of $30 \mathrm{mg}$ OD was reached for individual subjects in ARTS-DN, and the concentration-effect relationships were adequately captured up to these high exposures for all markers (ESM Figure S5).

The inclusion criteria and study design considered for the simulations are important for the predicted effect on all three markers, but especially for the prediction of the percentage of subjects with one or more serum potassium values $>5.5$ and $>6.0 \mathrm{mmol} / \mathrm{L}$. As previously discussed, the percentage of subjects exceeding the thresholds is highly dependent on the serum potassium concentration at baseline. It was indirectly assumed that reassessment at run-in and screening was not allowed, i.e., subjects with a serum potassium value $>4.8 \mathrm{mmol} / \mathrm{L}$ at run-in or screening were not included in the simulations. If reassessment is allowed considering pre-baseline, the percentages of subjects with one or more serum potassium values $>5.5$ and $>6.0 \mathrm{mmol} / \mathrm{L}$ are expected to be higher.

Simulations indicate that the expected changes in UACR, serum potassium concentration, and eGFR are relatively small when increasing the dose from $20 \mathrm{mg}$ OD to $30 \mathrm{mg}$ OD, with $6,0.4$, and 0.5 percentage points more subjects reaching the respective thresholds.

\subsection{Japanese Patients Versus Patients Enrolled in the Global Study}

The developed pharmacokinetic and PK/PD models were applied to, and updated based on, the combined data from the global and Japanese studies (Table 2). The exposure in Japanese patients was $0.4 \%$ higher than in the global population, and the median $C_{\max }$ was $7.4 \%$ higher in Japanese patients, but both distributions overlap with that of the global population. The differences were not significant in a two-sided Student $t$ test at $p<0.05$. No significant ethnic differences were observed in the PK/PD relationships for UACR, serum potassium concentration, or eGFR. Significance was decided based on a likelihood ratio test and applying a criterion of $p<0.001$. It should be noted that the sizes of the study populations were not based on power calculations specifically aimed at quantifying a population difference in pharmacokinetics and drug effect. However, $\mathrm{BW}$, which is a potential driver for differences in pharmacokinetics, was only found as a significant covariate on volume and not clearance.

\section{Conclusion}

The pharmacokinetics of finerenone and its effects on UACR, serum potassium concentration, and eGFR were adequately described by popPK and popPK/PD models in the ARTS-DN and ARTS-DN Japan populations. Model analyses indicate that the pharmacological effects on reducing the efficacy marker UACR, as well as effects on safety parameters increasing serum potassium concentration and decreasing eGFR, generally approach saturation or attenuate towards the highest dose of $20 \mathrm{mg}$ OD investigated in ARTS-DN. Simulations demonstrated that increasing the dose beyond $20 \mathrm{mg}$ OD to $30 \mathrm{mg}$ OD would result in relatively small additional increases in the percentage of subjects reaching efficacy targets and safety thresholds. It is difficult to evaluate to what degree a small incremental decrease in UACR would translate into clinically meaningful benefit, also considering potentially counteracting effects on clinical efficacy from increased serum potassium and decreased eGFR, and in addition the potential decrease in tolerability in progressive kidney disease. Doses of both 10 and $20 \mathrm{mg}$ OD appeared safe and efficacious at reducing albuminuria. PopPK/PD models are in place to investigate the dose-exposure-response relationship in phase III for relevant efficacy and safety parameters and link these to clinical outcomes.

Acknowledgements Medical writing assistance was provided by Amie Hedges (Oxford PharmaGenesis Ltd, Oxford, UK), funded by Bayer AG. The analysis was kindly supported by Drs. Kirsten Bergmann, Oliver Ackaert, Eline van Maanen, and Jean Smeets from LAP\&P, funded by Bayer AG, as well as Dr. Andreas Seelmann and Nicholas Downie, Bayer AG. The authors would like to thank Drs. Alain Gay and Christina Nowack, Bayer AG, for constructive comments during manuscript preparation. 


\section{Compliance with Ethical Standards}

Funding This publication and the work involved were funded by Bayer AG.

Conflict of interest NS and HJD were paid consultants for Bayer AG during the conduct of the analysis. RH, AJ, PK, JL, DG, BP, and TE are employed by Bayer AG. RH, AJ, JL, DG, BP, and TE are Bayer AG shareholders.

Open Access This article is distributed under the terms of the Creative Commons Attribution-NonCommercial 4.0 International License (http://creativecommons.org/licenses/by-nc/4.0/), which permits any noncommercial use, distribution, and reproduction in any medium, provided you give appropriate credit to the original author(s) and the source, provide a link to the Creative Commons license, and indicate if changes were made.

\section{References}

1. Navaneethan SD, Nigwekar SU, Sehgal AR, Strippoli GF. Aldosterone antagonists for preventing the progression of chronic kidney disease: a systematic review and meta-analysis. Clin J Am Soc Nephrol. 2009;4(3):542-51.

2. Barfacker L, Kuhl A, Hillisch A, Grosser R, Figueroa-Perez S, Heckroth H, et al. Discovery of BAY 94-8862: a nonsteroidal antagonist of the mineralocorticoid receptor for the treatment of cardiorenal diseases. ChemMedChem. 2012;7(8):1385-403.

3. Kolkhof P, Delbeck M, Kretschmer A, Steinke W, Hartmann E, Barfacker L, et al. Finerenone, a novel selective nonsteroidal mineralocorticoid receptor antagonist protects from rat cardiorenal injury. J Cardiovasc Pharmacol. 2014;64(1):69-78.

4. Pitt B, Kober L, Ponikowski P, Gheorghiade M, Filippatos G, Krum H, et al. Safety and tolerability of the novel non-steroidal mineralocorticoid receptor antagonist BAY 94-8862 in patients with chronic heart failure and mild or moderate chronic kidney disease: a randomized, double-blind trial. Eur Heart J. 2013;34(31):2453-63.

5. Bakris GL, Agarwal R, Chan JC, Cooper ME, Gansevoort RT, Haller $\mathrm{H}$, et al. Effect of finerenone on albuminuria in patients with diabetic nephropathy: a randomized clinical trial. JAMA. 2015;314(9):884-94.

6. Katayama S, Yamada D, Nakayama M, Yamada T, Myoishi M, Kato M, et al. A randomized controlled study of finerenone versus placebo in Japanese patients with type 2 diabetes mellitus and diabetic nephropathy. J Diabetes Complic. 2017;31(4):758-65.

7. Lentini S, Heinig R, Kimmeskamp-Kirschbaum N, Wensing G. Pharmacokinetics, safety and tolerability of the novel, selective mineralocorticoid receptor antagonist finerenone - results from first-in-man and relative bioavailability studies. Fundam Clin Pharmacol. 2016;30(2):172-84.

8. Heinig R, Kimmeskamp-Kirschbaum N, Halabi A, Lentini S. Pharmacokinetics of the novel nonsteroidal mineralocorticoid receptor antagonist finerenone (BAY 94-8862) in individuals with renal impairment. Clin Pharmacol Drug Dev. 2016;5(6):488-501.

9. Heinig R, Lambelet M, Nagelschmitz J, Alatrach A, Halabi A. Pharmacokinetics of the novel nonsteroidal mineralocorticoid receptor antagonist finerenone (BAY 94-8862) in individuals with mild or moderate hepatic impairment. Eur J Drug Metab Pharmacokinet. 2019. https://doi.org/10.1007/s13318-019-00547-x.
10. Gerisch M, Heinig R, Engelen A, Lang D, Kolkhof P, Radtke M, et al. Biotransformation of finerenone, a novel nonsteroidal mineralocorticoid receptor antagonist, in dogs, rats, and humans, in vivo and in vitro. Drug Metab Dispos. 2018;46(11):1546-55.

11. Heinig R, Gerisch M, Engelen A, Nagelschmitz J, Loewen S. Pharmacokinetics of the Novel, selective, non-steroidal mineralocorticoid receptor antagonist finerenone in healthy volunteers: results from an absolute bioavailability study and drug-drug interaction studies in vitro and in vivo. Eur J Drug Metab Pharmacokinet. 2018;43(6):715-27.

12. Heerspink HJ, Kropelin TF, Hoekman J, de Zeeuw D, Reducing Albuminuria as Surrogate Endpoint (REASSURE) Consortium. Drug-induced reduction in albuminuria is associated with subsequent renoprotection: a meta-analysis. J Am Soc Nephrol. 2015;26(8):2055-64.

13. Carrero JJ, Grams ME, Sang Y, Arnlov J, Gasparini A, Matsushita K, et al. Albuminuria changes are associated with subsequent risk of end-stage renal disease and mortality. Kidney Int. 2017;91(1):244-51.

14. Coresh J, Heerspink HJL, Sang Y, Matsushita K, Arnlov J, Astor $\mathrm{BC}$, et al. Change in albuminuria and subsequent risk of end-stage kidney disease: an individual participant-level consortium metaanalysis of observational studies. Lancet Diabetes Endocrinol. 2019;7(2):115-27.

15. Heerspink HJL, Greene T, Tighiouart H, Gansevoort RT, Coresh $\mathrm{J}$, Simon AL, et al. Change in albuminuria as a surrogate endpoint for progression of kidney disease: a meta-analysis of treatment effects in randomised clinical trials. Lancet Diabetes Endocrinol. 2019;7(2):128-39.

16. Jamsen KM, McLeay SC, Barras MA, Green B. Reporting a population pharmacokinetic-pharmacodynamic study: a journal's perspective. Clin Pharmacokinet. 2014;53(2):111-22.

17. Ruilope LM, Agarwal R, Chan JC, Cooper ME, Gansevoort RT, Haller H, et al. Rationale, design, and baseline characteristics of ARTS-DN: a randomized study to assess the safety and efficacy of finerenone in patients with type 2 diabetes mellitus and a clinical diagnosis of diabetic nephropathy. Am J Nephrol. 2014;40(6):572-81.

18. Levey AS, Coresh J, Greene T, Stevens LA, Zhang YL, Hendriksen $S$, et al. Using standardized serum creatinine values in the Modification of Diet in Renal Disease study equation for estimating glomerular filtration rate. Ann Intern Med. 2006;145(4):247-54.

19. Matsuo S, Imai E, Horio M, Yasuda Y, Tomita K, Nitta K, et al. Revised equations for estimated GFR from serum creatinine in Japan. Am J Kidney Dis. 2009;53(6):982-92.

20. Chudleigh RA, Ollerton RL, Dunseath G, Peter R, Harvey JN, Luzio S, et al. Performance of the revised '175' Modification of Diet in Renal Disease equation in patients with type 2 diabetes. Diabetologia. 2008;51(9):1714-8.

21. Levey AS, Stevens LA, Schmid CH, Zhang YL, Castro AF 3rd, Feldman HI, et al. A new equation to estimate glomerular filtration rate. Ann Intern Med. 2009;150(9):604-12.

22. Horio M, Imai E, Yasuda Y, Watanabe T, Matsuo S. Modification of the CKD Epidemiology Collaboration (CKD-EPI) equation for Japanese: accuracy and use for population estimates. Am J Kidney Dis. 2010;56(1):32-8.

23. Grune J, Beyhoff N, Smeir E, Chudek R, Blumrich A, Ban Z, et al. Selective mineralocorticoid receptor cofactor modulation as molecular basis for finerenone's antifibrotic activity. Hypertension. 2018;71(4):599-608.

24. de Zeeuw D, Remuzzi G, Parving HH, Keane WF, Zhang Z, Shahinfar S, et al. Proteinuria, a target for renoprotection in patients with type 2 diabetic nephropathy: lessons from RENAAL. Kidney Int. 2004;65(6):2309-20. 
25. de Zeeuw D, Remuzzi G, Parving HH, Keane WF, Zhang Z, Shahinfar S, et al. Albuminuria, a therapeutic target for cardiovascular protection in type 2 diabetic patients with nephropathy. Circulation. 2004;110(8):921-7.

26. Weir MR. Acute fall in glomerular filtration rate with renin-angiotensin system inhibition: a biomeasure of therapeutic success? Kidney Int. 2011;80(3):235-7.
27. Holtkamp FA, de Zeeuw D, Thomas MC, Cooper ME, de Graeff PA, Hillege HJ, et al. An acute fall in estimated glomerular filtration rate during treatment with losartan predicts a slower decrease in long-term renal function. Kidney Int. 2011;80(3):282-7. 